\title{
RESEARCH
}

\section{Adipose tissue-derived autotaxin causes cardiomyopathy in obese mice}

\author{
Yousheng Xu1,2, Yongshun Wang1,2, Jingjin Liư1,2, Wei Cao1,2, Lili Li1,2, Hongwei Du1,2, Enbo Zhan 1,2, Ruoxi Zhang1,2, \\ Huimin Liu' ${ }^{1,2}$, Maoen $\mathrm{Xu} \mathbf{u}^{1,2}$, Tao Chen ${ }^{1,2}$, Yilin $\mathrm{Qu}^{3}$ and Bo $\mathrm{Yu}^{1,2}$ \\ 1Department of Cardiology, The Second Affiliated Hospital of Harbin Medical University, Harbin, Heilongjiang, China \\ 2The Key Laboratory of Myocardial Ischemia, Chinese Ministry of Education, Harbin, Heilongjiang, China \\ ${ }^{3}$ Department of Biochemistry and Molecular Biology, Harbin Medical University, Harbin, Heilongjiang, China
}

Correspondence should be addressed to Y Qu or B Yu: quyilin1982@163.com or yubodr@163.com

\begin{abstract}
The prevalence of obesity is dramatic increased and strongly associated with cardiovascular disease. Adipokines, secreted from adipose tissues, are critical risk factors for the development of cardiomyopathy. Present study aimed to investigate the pathophysiological role of autotaxin in obesity-related cardiomyopathy. In high-fat dietfed mice, autotaxin was mainly synthesized and secreted from adipocytes. The increased accumulation of cardiac autotaxin was positively associated with cardiac dysfunction in obese mice. Interestingly, specific blockage of adipose tissue autotaxin effectively protected against high-fat diet-induced cardiac structural disorders, left ventricular hypertrophy and dysfunction. Inhibition of autotaxin further improved high-fat dietinduced cardiac fibrosis and mitochondrial dysfunction, including improvement of mitochondrial structure, mass and activities. Our findings demonstrated intervention of adipose tissue biology could influence cardiac modification in obese mice, and adipocytederived autotaxin was a potential diagnostic marker and therapeutic target for obesityrelated cardiomyopathy.
\end{abstract}

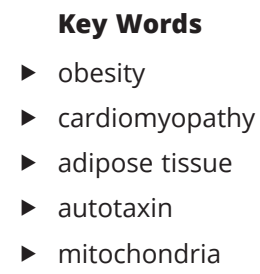

Journal of Molecular Endocrinology (2019) 63, 113-121

\section{Introduction}

The prevalence of overweight and obesity in the world has risen dramatically. Obesity is associated with significant metabolic disorders and may trigger severe cardiovascular diseases (Piche et al. 2018). Among them, cardiomyopathy, exhibiting as cardiac structural remodeling and dysfunction in terms of abnormal energy metabolism, myocardial inflammation and impaired left ventricular contractility, leads to unhealthy outcome in obese patients (Alpert et al. 2014). Pathological cardiac remodeling, usually chartered as reduced ejection fraction and fractional shortening, eventually transfers to heart failure (Alpert et al. 2014). Therefore, there is an urgent need to understand the pathological process of cardiomyopathy, which will lead to explore novel therapeutic approaches for alleviating cardiac injuries and preventing detrimental outcomes.

Adipose tissue, mainly comprising neutral lipid, is closely correlated with amounts of clinical parameters such as systemic insulin sensitivity, glucose tolerance and serum lipid profiles (Janssen et al. 2002). Visceral adipose tissue mass is more closely correlated with obesity-associated metabolic syndromes (DiPietro et al. 1999). The elevated fat mass in humans is an independent risk factor for myocardial infarction, stroke and type 2 diabetes mellitus 
(Hubert et al. 1983, Kurth et al. 2002). Adipocytes can secrete a number of factors, named adipokines, into other tissues, and participate in a complex network of obesityrelated cardiovascular disease. For example, adiponectin, mainly secreted from white adipose tissue, protected against dietary- or ischemia-induced cardiac injuries by binding to its receptors in the heart (Shibata et al. 2005, Tamura et al. 2007). Vaspin, secreted from visceral fat, also exhibited cardiac protective benefits in obese models (Nakatsuka et al. 2013). On the contrast, adipocyte fatty acid-binding protein (A-FABP)-mediated cardiovascular inflammation and cardiac dysfunction (Zhou et al. 2015). These findings supported that adipocytes could modulate cardiac function by adipokines in obese status.

Autotaxin, encoded by ENPP2 gene in human, is widely expressed in different tissues, including adipose tissue, kidney and pancreas (Rancoule et al. 2014). Previous study showed that the expression of autotaxin was drastically increased during the differentiation of preadipocyte into mature adipocytes (Ferry et al. 2003). Consistently, adipocyte autotaxin mRNA levels were increased by about four-fold in $\mathrm{db} / \mathrm{db}$ mice when compared to lean littermates (Boucher et al. 2005). In contrast, depletion of autotaxin could protect against high-fat diet (HFD)-induced obesity, insulin resistance and glucose intolerance in obese mice (Nishimura et al. 2014). A study identified that autotaxin was involved in the pathobiology of cardiovascular disease (Bouchareb et al. 2015). Upregulation of lysophosphatidic acid (LPA), whose production was controlled by autotaxin, induced cardiomyocyte hypertrophy (Hilal-Dandan et al. 2004). However, whether autotaxin participates in the process of obesity-related cardiomyopathy is still unknown.

To investigate the potential biological role of autotaxin in the process of obesity-related cardiomyopathy, we utilized an obese mouse model to determine the effects of autotaxin on cardiac pathophysiology. Our findings supported autotaxin, mainly secreted from adipocytes, mediated cardiac injuries in obese mice, but blockage of adipose tissue autotaxin effectively improved cardiac structural disorders and mitochondrial function.

\section{Materials and methods}

\section{Reagents}

ELISA kits for detecting autotaxin (\#LS-F16526) and LPA (\#LS-F25111) were purchased from LSBio (Lifespan Biosciences, WA, USA). The Citrate Synthase Activity
Colorimetric Assay Kit (\#K318) was purchased from Biovision (Biovision, CA, USA). The lentivirus encoding adiponectin promoter-derived autotaxin siRNA or control siRNA was gifted from Dr Lin Zhu (Wuhan University). Hematoxylin, eosin solution and Sirius red staining kit were purchased from Sigma chemicals. Anti-autotaxin antibody (\#ab137590) was from Abcam. Anti-TGF- $\beta 1$ (\#3711), anti-Smad3 (\#9513), anti- $\alpha$-porin (\#4866) and anti- $\beta$-Tubulin (\#2146) antibodies were from Cell Signaling Technology.

\section{Animal experiment}

Six-week-old age male C57BL/6J mice were randomly assigned to standard chow (\#5001, LabDiet) providing $11.4 \%$ calories from fat or HFD (\#D12492, Research Diets) with $60 \%$ calories from fat for $0,8,16$ and 32 weeks. Another set of animal study included obese mice fed with HFD for 8-week were locally injected with $1.2 \times 10^{9}$ lentivirus particles encoding autotaxin siRNA or control siRNA into two sides of mouse epididymal adipose tissue. These mice were fed HFD for another 8 weeks. Then the mice were killed, and the serum and cardiac tissue were collected for further analysis. The experimental procedure described here was approved by the Institutional Animal Use and Care Committee at the Harbin Medical University (Harbin, China).

\section{Echocardiography analysis}

Cardiac geometry and functional parameters were recorded in anesthetized mice using echocardiographic examinations which were obtained by using the Vevo 2100 system (Vevo). Anterior and posterior wall thicknesses and diastolic and systolic left ventricular dimensions were recorded from M-mode images. Echocardiographic parameters included ejection fraction $(\mathrm{EF})$, fraction shorten (FS), heart rat, left ventricular anterior wall (LVAW), left ventricular internal dimension (LVID), left ventricle posterior wall thickness (LVPW) and LV mass.

\section{Cardiac histological analysis}

Mouse hearts were fixed in $4 \%$ paraformaldehyde for $24 \mathrm{~h}$ and embedded in paraffin. Five micrometer paraffin sections were prepared and stained with hematoxylin and eosin solution or Sirius red staining kit. To measure the histological changes, the cardiac images were observed under a light microscope (Nikon). 
Total RNA extraction, cDNA synthesis, reverse transcription and real-time PCR

The total RNA was homogenized in TRIzol and isolated from mouse hearts or primary cardiomyocytes according to the manufacturer's protocol. Reverse transcription was performed using the Superscript III Reverse Transcription System (Invitrogen), and real-time PCR analysis was performed using SYBR Green (Applied Biosystems). The sequence of primers were listed as following: Autotaxin: F-GACCCTAAAGCCATTATTGCTAA; R-GGGAAGGTGCTGTTTCATGT, Gapdh: F-AGGAGCG AGACCCCACTAAC; R-GATGACCCTTTTGGCTCCAC. Relative Autotaxin level was normalized to Gapdh level.

\section{Measurement of serum autotaxin and LPA}

The mouse circulating levels of autotaxin and LPA were detected by ELISA kits from LSBio (Lifespan Biosciences) according to the manufacture's instruction.

\section{Western blot analysis}

Protein extracts $(50 \mu \mathrm{g})$ were run on $10 \%$ SDS-PAGE. The protein was then transferred to a PVDF membrane (Amersham Biosciences). The membrane was blocked for $1 \mathrm{~h}$ at room temperature with $10 \%$ milk in phosphate-buffered saline/0.05\% Tween 20. The blots were incubated overnight at $4^{\circ} \mathrm{C}$ with antiautotaxin, anti-TGF- $\beta 1$, anti-Smad3 or anti-Tubulin antibody and secondary antibody (Cell Signaling). The protein expression was visualized using enhanced chemiluminescence reagents (Bio-Rad). The amounts of the proteins were analyzed using Image $\mathrm{J}$ analysis software, version $1.38 \mathrm{e}$.

\section{Mitochondrial functional analysis}

The cardiac citric synthase activity was analyzed by Citrate Synthase Activity Colorimetric Assay Kit (Biovision). The cardiac ATP was measured using an ATP measurement kit (Molecular Probes). The endogenous basal oxygen consumption was measured with a clark electrode in a water-jacketed chamber connected to a circulating water bath (Hansatech, Norfolk, UK). For enzyme activity of mitochondrial complex I, mitochondria were isolated and measured according to the method described in previous report (Nie et al. 2017, 2018). The complex I activity was expressed as a ratio to citrate synthase activity to account for mitochondrial enrichment.

\section{Statistical analysis}

Data were presented as mean \pm s.E.M. The one-way ANOVA was used for comparing three groups, and Students't-test was used for comparing two groups. GraphPad Prism 7 (GraphPad) was used to analyze the statistical significance between sets of data. Differences were considered to be significant at $P<0.05$.

\section{Results}

\section{Adipose tissue is the main source of autotaxin production during obesity}

Autotaxin is involved in amounts of metabolic diseases, but the distribution of autotaxin is still unclear during obese process. Here, we firstly measured Autotaxin mRNA levels in different tissues, including visceral adipose tissue, heart, brain and kidney. As showed in Fig. 1A, adipose tissue had higher mRNA level of Autotaxin in both lean and obese mice, as compared with other tissues. HFD increased Autotaxin to more than four-fold to lean mice in visceral adipose tissue, but not affected mRNA levels
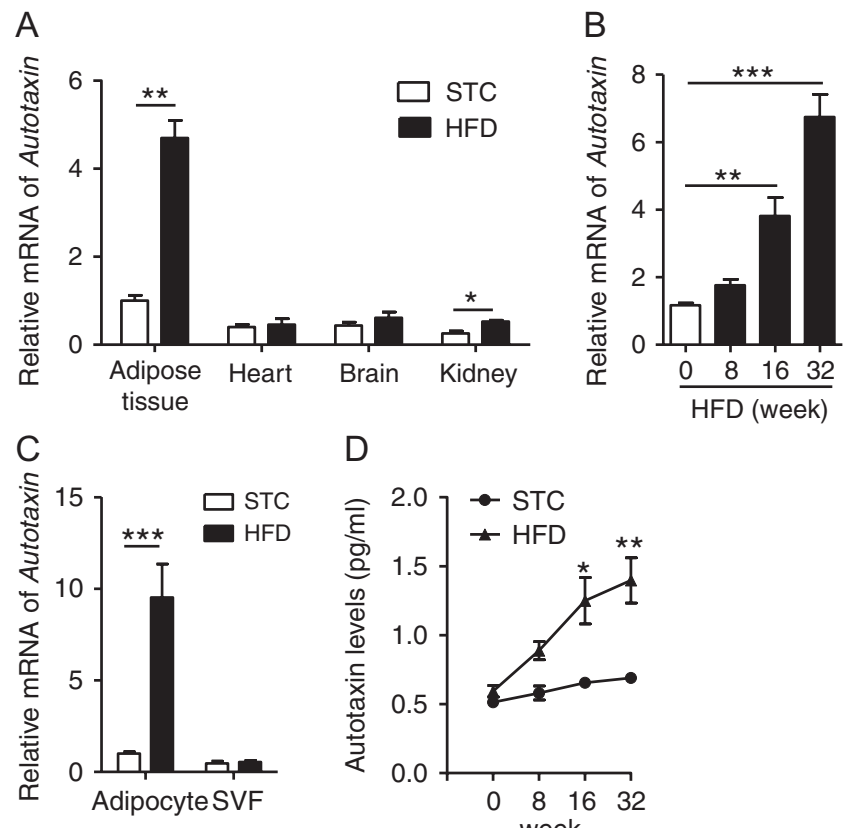

$\mathrm{D}$

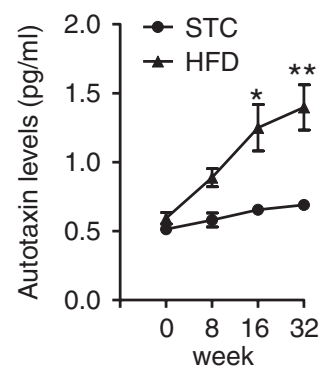

\section{Figure 1}

The elevation of autotaxin levels during obesity. Six-week, male C57BL/6] mice were fed with standard chow (STC) or high-fat diet (HFD) for 0, 8, 16 and 32 weeks. (A) The mRNA levels of Autotaxin in different tissues after STC or HFD feeding for 16 weeks. (B) The changes of Autotaxin mRNA levels during obesity in visceral adipose tissues. (C) The Autotaxin levels in mature adipocytes and stromal vascular fraction (SVF). (D) The circulating levels of autotaxin. Data are shown as mean \pm S.E.M. $\left({ }^{*} P<0.05, * * P<0.01\right.$, $\star * \star P<0.001, n=6)$. 
in heart or brain (Fig. 1A). Furthermore, HFD also time dependently increased Autotaxin level in adipose tissues (Fig. 1B). Then, we isolated and measured Autotaxin levels in mature adipocytes and stromal vascular fraction (SVF) in visceral adipose tissue. Adipocytes had much higher levels of Autotaxin than SVF in both lean and obese mice (Fig. 1C). More interestingly, circulating level of autotaxin was also elevated during obesity (Fig. 1D). Taken together, adipose tissue mainly synthesized and secreted autotaxin in high-fat diet-fed mice.

\section{Cardiac autotaxin is closely associated with cardiac function in obese mice}

Although mRNA level of cardiac Autotaxin was lower, we found the autotaxin protein levels were obviously increased during HFD treatment. As showed in Fig. 2A and $\mathrm{B}$, lean mouse heart had almost undetectable level of autotaxin, but HFD significantly upregulated autotaxin protein level after 8- or 16-week feeding.

Next, we measured the changes of cardiac parameters, including $\mathrm{EF}$ and FS. As showed in Fig. 2C and D, HFD remarkably decreased the values of EF (C) and FS (D), as compared with lean mice. To investigate the possible links between cardiac autotaxin and functional parameters, we further analyzed the potential correlation. As showed in Fig. 2E and F, cardiac autotaxin level was negatively correlated with EF $(r=-0.5904, P<0.05)$ and FS $(r=-0.8423$, $P<0.01)$. All these findings indicated that adipose tissue autotaxin might have entered into cardiac tissues during obesity and impaired mouse cardiac function.

\section{Adipose tissue-specific deficiency of autotaxin improves high-fat diet-induced cardiac injuries}

To address the crosstalk between adipose tissue and heart, we generated specific adipose autotaxin-deficient mice by local injection with lentivirus-encoding Autotaxin siRNA. C57BL/6J mice were fed with HFD for 8 weeks, and $1.2 \times 10^{9}$ particles of lentivirus-encoding autotaxin siRNA (siATX) or control siRNA (siCtrl) were locally injected into visceral adipose tissue. After HFD feeding for 8 weeks, we firstly measured the expression levels of autotaxin in adipose tissues. Epididymal adipose tissues injected with siATX exhibited significantly lower level of autotaxin, as compared with siCtrl-treated mice (Fig. 3A and B). Similarly, as showed in Fig. 3C, lentivirus-encoding siATX significantly decreased circulating autotaxin level to $30 \%$ of siCtrl-treated obese mice $(P<0.001)$. LPA, produced from lysophosphatidylcholine (LPC) by autotaxin, also affected cardiac function. Our results found HFD treatment also increased circulating LPA level $(P<0.01)$, but adipose tissue deficiency of autotaxin almost reversed the upregulation in obese mice (Fig. 3D). siATX also decreased autotaxin accumulation in obese cardiac tissues (Fig. 3E and F).

Next, we found HFD increased body weight, but siATX did not affect the body weight gain (Fig. 3G). However, autotaxin deficiency decreased HFD-induced
A

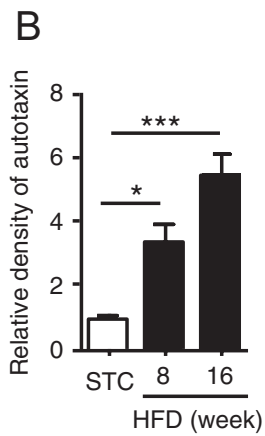

C

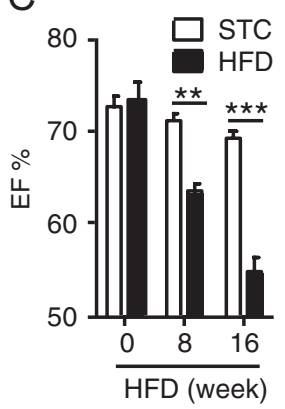

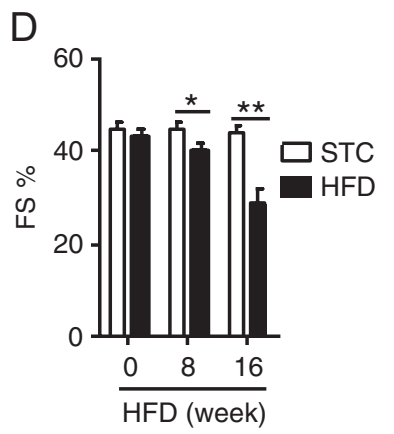

E

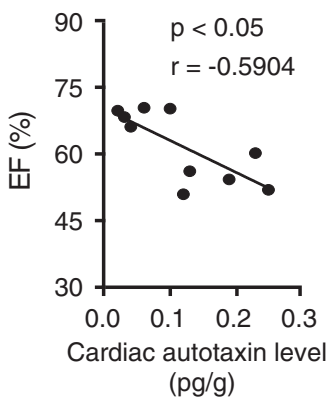

$\mathrm{F}$

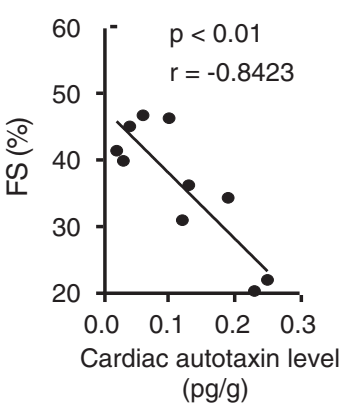

Figure 2

The cardiac autotaxin level is positively associated with high-fat diet-induced cardiac dysfunction. Six-week, male C57BL/6J mice were fed with STC or HFD for 0,8 and 16 weeks. (A and B) Western blot analysis of cardiac autotaxin levels (A) and quantitative analysis of relative density (B). (C and D) Ultrasound analysis of cardiac parameters ejection fraction $(E F, C)$ and fractional shortening (FS, D). (E and F) The correlation between cardiac autotaxin level and EF (E) or FS (F). Data are shown as mean \pm S.E.M. $\left({ }^{*} P<0.05, * \star P<0.01\right.$, $\star \star \star P<0.001, n=6)$. 

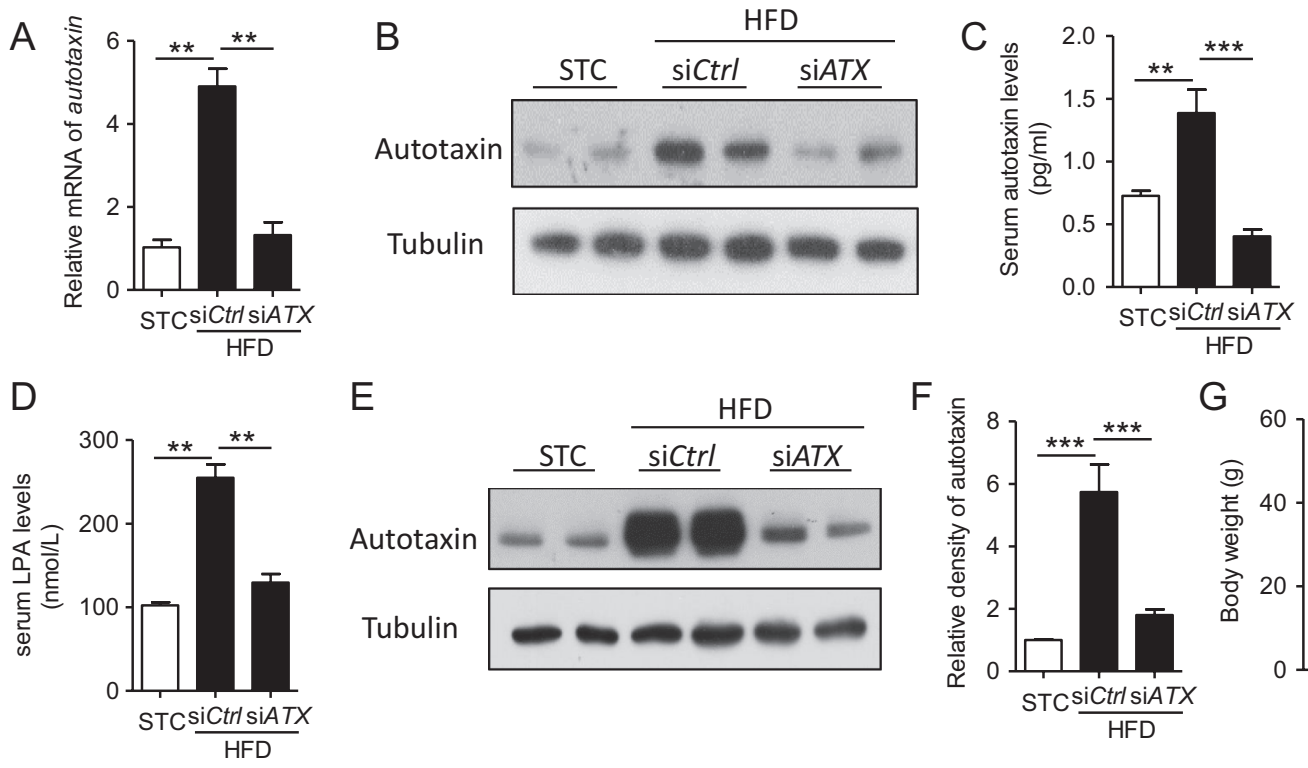

$\mathrm{E}$
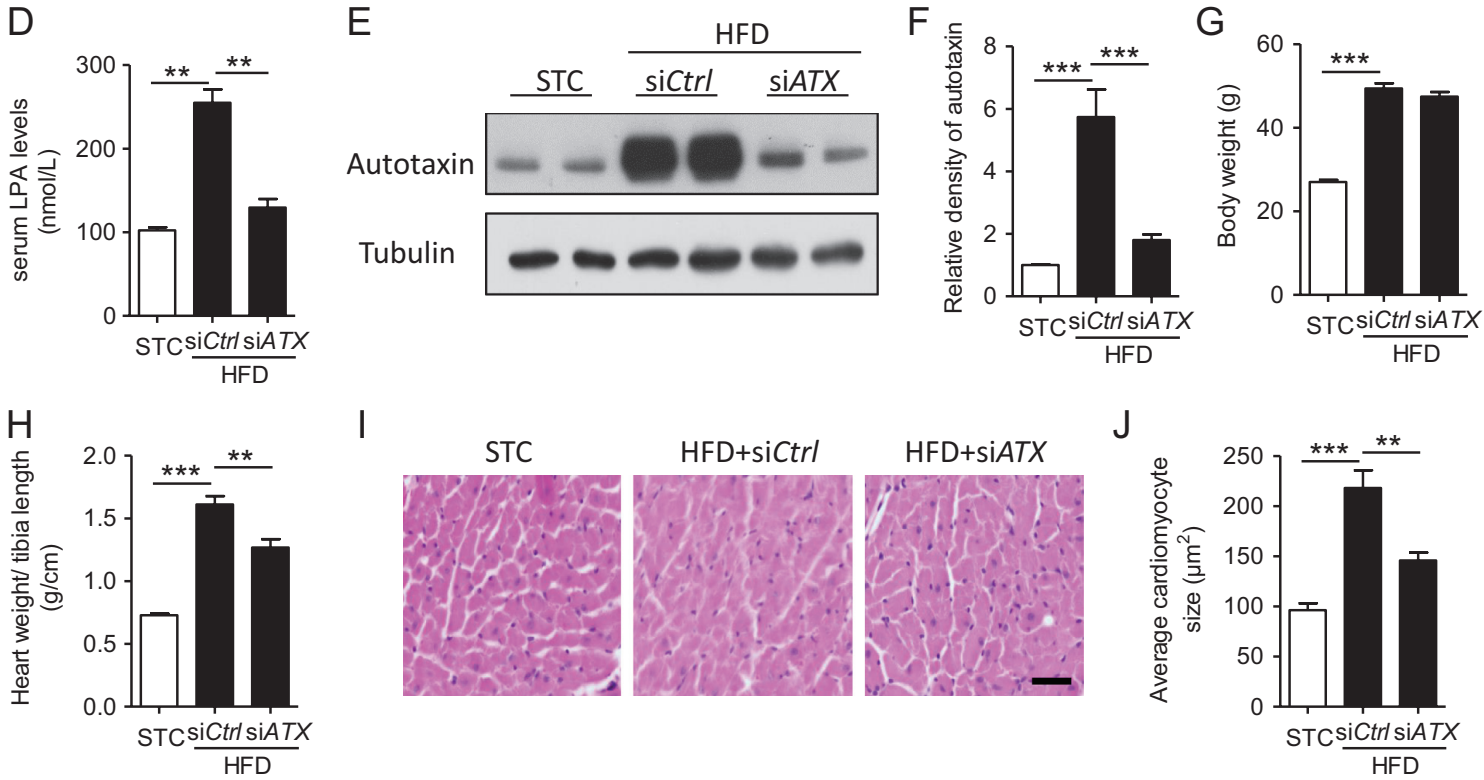

I
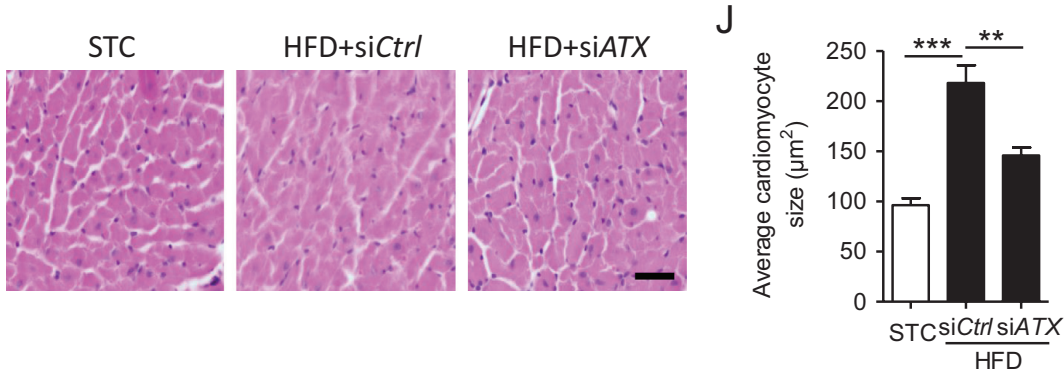

\section{Figure 3}

Blockage of adipose autotaxin protected against high-fat diet-induced cardiac structural disorders. Six-week, male C57BL/6J mice were fed with HFD for 8 weeks, and locally injected with $1.2 \times 10^{9}$ particles of lentivirus-encoding autotaxin siRNA (siATX) or control siRNA (siCtr) into visceral adipose tissue. After HFD feeding for 8 weeks, the visceral adipose tissues, serum and heart tissues were collected for analysis. (A and B) The adipose autotaxin levels were measured by RT-qPCR (A) and Western blot (B). (C and D) The circulating levels of autotaxin (C) and LPA (D). (E and F) Western blot analysis of cardiac autotaxin levels (E) and quantitative analysis of the relative density (F). (G, H and I) Body weight (G), heart weight/tibia length (H) and cardiac H\&E staining (I). Scale bar: $40 \mu \mathrm{m}$. () Average size of cardiomyocytes. Data are shown as mean \pm s.E.M. ( $\left.{ }^{\star *} P<0.01, \star \star \star P<0.001, n=6\right)$. A full colour version of this figure is available at https://doi.org/10.1530/JME-18-0242.

cardiac hypertrophy, exhibited as decreasing values of heart weight/tibia length (Fig. $3 \mathrm{H}$ ), left ventricular wall thickness, left ventricular dimension and left ventricular mass (Table 1). Furthermore, H\&E staining also found silence of adipose autotaxin effectively improved cardiac structural abnormalities (Fig. 3I). In detail, the autotaxin deficiency attenuated HFD-induced size enlargement of cardiomyocytes, as siCtrl-treated obese mice (Fig. 3J, $P<0.01$ ).

\section{Adipose tissue autotaxin deficiency decreases high-fat diet-induced cardiac fibrosis and mitochondrial dysfunction}

Cardiac fibrosis is one of the key cardiac characteristics in obese mice and human subjects (Kawano et al. 2000,
Tamura et al. 2000). To this end, we further investigated the effects of autotaxin on cardiac fibrosis. The Sirius red staining showed that siATX almost completely decreased collagen deposit in obese cardiac tissues (Fig. 4A and B, $P<0.001)$. TGF- $\beta 1 /$ Smad signaling determines cardiac fibrosis in obese models (Liu et al. 2018). Similarly, HFD upregulated the expression of TGF- $\beta 1$ and Smad3, as compared with lean mice (Fig. 4C and E). However, siATX could significantly decrease these protein levels.

Heart possesses abundant of mitochondria, which support the cardiac energy expenditure (Chen \& Lesnefsky 2015). Consistent with previous studies, we also found HFD damaged the cardiac mitochondrial morphology (Fig. 5A), but siATX improved the mitochondrial structure. The decreased level of mitochondrial marker, $\alpha$-porin, was also significantly increased in siATX-treated obese 
Table 1 Echocardiographic analysis of mouse cardiac parameters.

Heart rate (beats/min)
LVAW;d (mm)
LVAW;s (mm)
LVID;d (mm)
LVID;s (mm)
LVPW;d (mm)
LVPW;s (mm)
LV Mass (mg)

\begin{tabular}{c}
\hline STC \\
\hline $467 \pm 27^{* *}$ \\
$0.86 \pm 0.11^{* * *}$ \\
$1.22 \pm 0.03^{\star * *}$ \\
$3.81 \pm 0.14^{*}$ \\
$2.26 \pm 0.19^{*}$ \\
$0.69 \pm 0.11^{* * *}$ \\
$1.20 \pm 0.11^{*}$ \\
$103.86 \pm 5.03^{\star * *}$ \\
\hline
\end{tabular}

\begin{tabular}{c}
\hline HFD + siCtrI \\
\hline $511 \pm 39$ \\
$0.99 \pm 0.09$ \\
$1.51 \pm 0.21$ \\
$4.12 \pm 0.21$ \\
$2.68 \pm 0.23$ \\
$0.96 \pm 0.10$ \\
$1.28 \pm 0.08$ \\
$159.45 \pm 9.65$ \\
\hline
\end{tabular}

\begin{tabular}{c}
\hline HFD + siATX \\
\hline $495 \pm 21$ \\
$0.89 \pm 0.11 * *$ \\
$1.35 \pm 0.09 * *$ \\
$3.94 \pm 0.16$ \\
$2.52 \pm 0.19$ \\
$0.82 \pm 0.15 * *$ \\
$1.22 \pm 0.13$ \\
$117.64 \pm 8.99 * * *$ \\
\hline
\end{tabular}

Data are shown as mean \pm S.E.M. ( ${ }^{*} P<0.05, * * P<0.01, * * * P<0.001$ as compared with HFD + siCtrl group, $n=6$ ).

d, diastolic; LV, left ventricle mass; LVAW, left ventricular anterior wall; LVID, left ventricular internal dimension; LVPW, left ventricle posterior wall thickness; s, systolic.

mice (Fig. 5B and C). Then, we measured the mitochondrial activities in mouse cardiac tissues. As showed in Fig. 5D, E, F and G, HFD decreased cardiac citric synthase activity (Fig. 5D), ATP production (Fig. 5E), oxygen consumption (Fig. 5F) and complex I activity (Fig. 5G), but siATX effectively blocked these downregulation. Consequently, HFD-induced triglyceride accumulation was obviously decreased in siATX-treated obese mice (Fig. 5H, $P<0.05$ ). All these results indicated adipose tissue autotaxin played a critical role in obesity-related cardiomyopathy.

\section{Discussion}

Autotaxin has been implicated in metabolic disorders including obesity and insulin resistance (Nishimura et al. 2014). However, the role of autotaxin in obesityrelated cardiomyopathy remains unknown. Specifically, it is unclear whether adipocyte-secreted autotaxin can influence cardiac pathophysiology. Current results showed that adipocytes were main sources of autotaxin production in obese mice. In addition, adipose tissuederived autotaxin could enter into heart and induce cardiac dysfunction. Specific silence of adipose tissue autotaxin protected against high-fat diet-induced cardiac structural disorders, fibrosis and mitochondrial dysfunction in obese mice. Taken together, this study suggested that autotaxin, mainly secreted from adipose tissue, mediated cardiac injuries.

Autotaxin is a secreted lysophospholipase D catalyzing the hydrolysis of lysophosphatidylcholine (LPC) to LPA, a pleiotropic growth factor-like phospholipid. Obese humans had higher mRNA level of autotaxin in adipose tissue and elevated circulating autotaxin, which were positively associated with metabolic disorders compared to those with normal metabolism (Rancoule et al. 2012). Furthermore, circulating levels of autotaxin were an independent predictor for non-alcoholic fatty liver disease (Reeves et al. 2015). Several obesity-related factors, such as interleukin-6 and glucose, induced the synthesis and secretion of autotaxin in obese mice
A
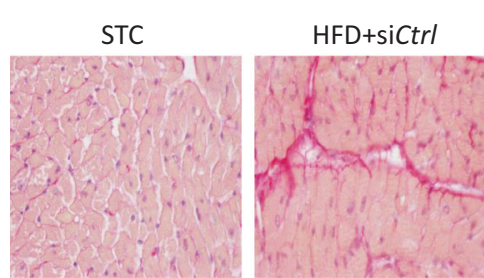

C

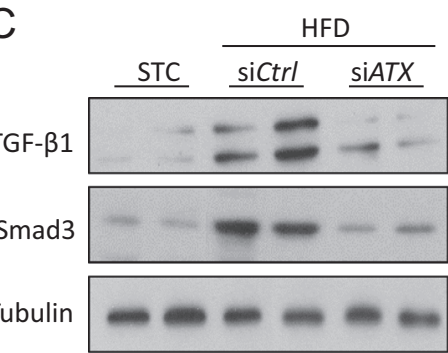

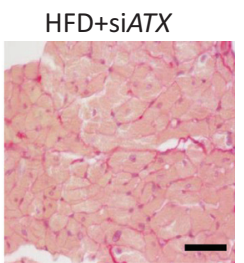

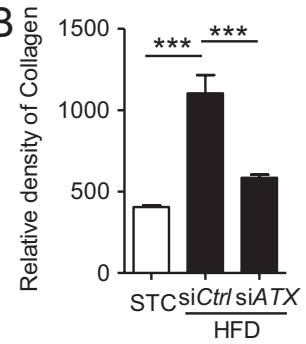

E

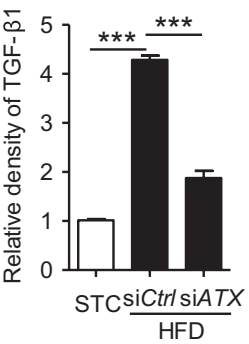

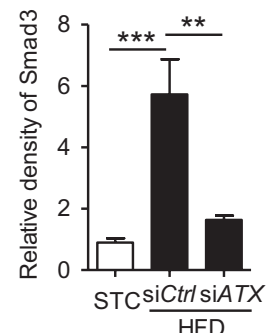

Figure 4

Blockage of adipose autotaxin attenuates high-fat diet-induced cardiac fibrosis. (A and B) Cardiac Sirius red staining for collagen $(A)$ and quantitative analysis of collagen content (B). Scale bar: $40 \mu \mathrm{m}$. (C, D and E) Western blot analysis of cardiac levels of TGF- $\beta 1$ and Smad3 (C) and quantitative analysis of relative density of protein expression ( $D$ and $E$ ). Data are shown as mean \pm S.E.M. $(* \star P<0.01, * \star * P<0.001, n=6)$. A full colour version of this figure is available at https:// doi.org/10.1530/JME-18-0242. 
A STC

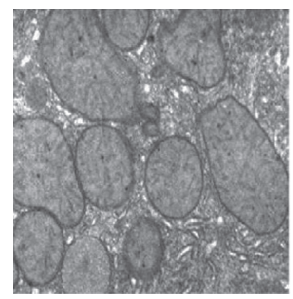

C

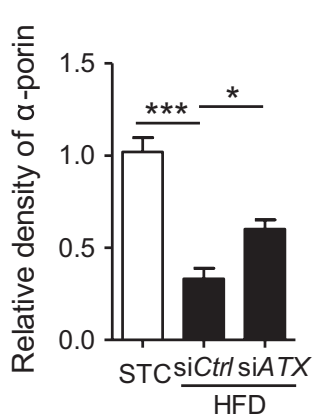

G

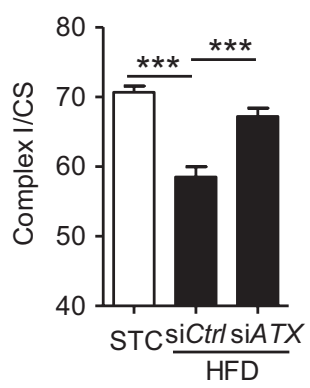

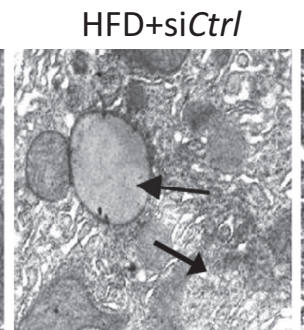

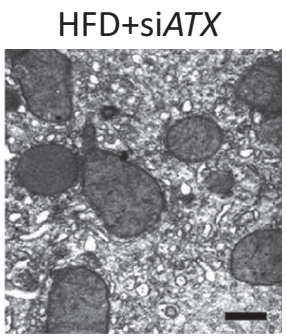

B

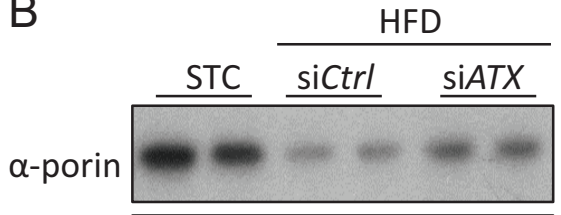

Tubulin

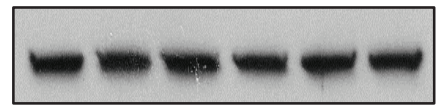

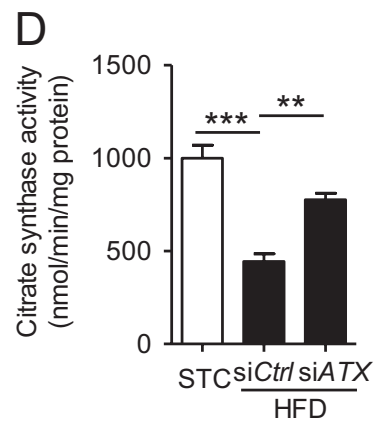

E

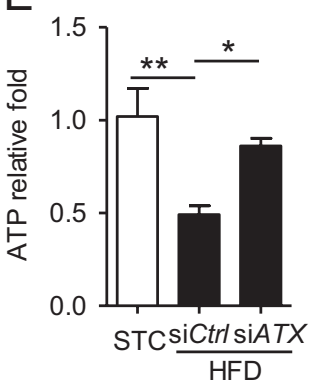

F

$\mathrm{H}$

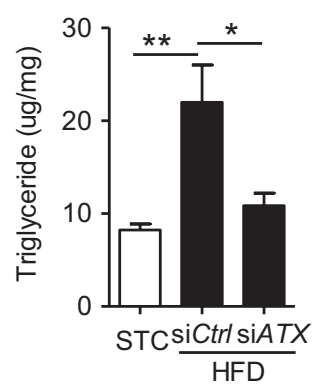

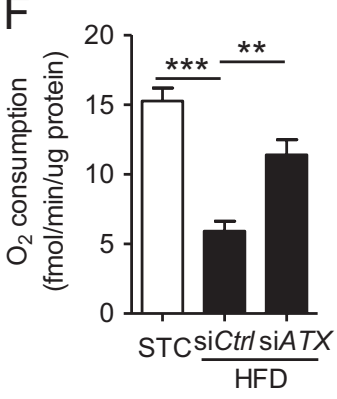

\section{Figure 5}

Blockage of adipose autotaxin improves cardiac mitochondrial expression and activities in obese mice. (A) Representative images of cardiac structure by electron microscope. (B and C) Western blot analysis of mitochondrial marker $\alpha$-porin level in hearts (B) and quantitative analysis of the relative density (C). (D, E, F and G) Measurement of cardiac citric synthase activity (D), ATP level (E), oxygen consumption (F) and mitochondrial complex I activity (G). (H) Biochemical analysis of cardiac triglyceride levels. Data are shown as mean \pm s.E.M. ( $* P<0.05, * \star P<0.01, \star \star \star P<0.001, n=6)$.

(D'Souza et al. 2017, Sun et al. 2017). Mechanistically, autotaxin had a number of effects in several cell types by binding to its $G$ protein-coupled receptors and mediated the development of several diseases (Yuelling \& Fuss 2008). There are several studies that found autotaxin participated in the process of cardiomyopathy. One study indicated that autotaxin was involved in the pathophysiology of cardiovascular disease (Bouchareb et al. 2015). Mechanistically, autotaxin signaling might induce cardiomyocyte hypertrophy by stimulating the activation of Rho-mediated signals in vitro (HilalDandan et al. 2004). A more recent finding addressed ATX-LPA pathway contributed to obesity-induced insulin resistance and metabolic disorders, including cardiac injuries (D'Souza et al. 2018). Consistently, current study also found that cardiac autotaxin participated in diet-induced cardiac dysfunction, hypertrophy, fibrosis and mitochondrial inactivation.

One of the key findings in the present study was to link the adipose tissue and heart by autotaxin. Adipose tissues, as an endocrine organ, released a complex set of proteinaceous factors acting on many other metabolically active tissues (Halberg et al. 2008). For example, leptin, as an archetypical adipokine, could enter into the brain and affected food intake and energy expenditure (Gautron \& Elmquist 2011). Adipocyte-secreted adiponectin had potent insulin-sensitizing, anti-apoptotic and antiinflammatory properties (Shetty et al. 2009). Adipose tissue-derived resistin also affected the metabolic profiles of peripheral tissues (Takeishi et al. 2007). Present study showed adipocyte-secreted autotaxin could enter into obese cardiac tissues and impaired cardiac EF and FS. 
Interestingly, specific depletion of adipose tissue autotaxin not only suppressed systemic and local autotaxin levels, but also protected against high-fat diet-induced cardiac injuries. These results supported that autotaxin was an important adipokine linking adipose tissues and heart, which provided a useful approach to intervene cardiomyopathy.

Although one recent study reported autotaxin signaling could induce cardiomyocyte hypertrophy in vitro (Hilal-Dandan et al. 2004), there was no animal or human study to address the effects of autotaxin on cardiac hypertrophy. Present findings showed blockage of autotatxin inhibited high-fat diet-induced cardiac hypertrophy, including reduction of heart weight, cell size, left ventricular dimension and left ventricular mass in obese mice. The LPA3/AKT/mTOR pathway was a potential signaling pathway in modulating cardiac hypertrophy (Yang et al. 2018). Consistent with the role of autotaxin in pulmonary fibrosis (Ninou et al. 2018), present study found autotaxin also participated in cardiac fibrosis. However, the underlying mechanisms in cardiac hypertrophy or fibrosis were still not fully disclosed. In the further research plan, we will explain the potential molecular mechanisms.

Another key finding was autotaxin might play important roles in regulating cardiac mitochondrial expression and activities. Mitochondria serve an important role in energy production and transfer to myocardial cells (Frisard \& Ravussin 2006). Mitochondrial abnormalities lead to severe cardiac dysfunction and structural disorders (Marin-Garcia et al. 2001). Among the mitochondrial complexes, mitochondrial complex I, as a main component of electron transfer chain (ETC), determines mitochondrial activity (Hirst 2013). Previous study has showed that global autotaxin deficiency improved mitochondrial pyruvate oxidation in mouse muscle (D'Souza et al. 2018). In the present study, electron microscope data demonstrated inhibition of cardiac autotaxin improved diet-induced mitochondrial morphological disorders. Western blot analysis and biochemical assays further supported autotaxin deficiency attenuated diet-induced reduction of mitochondrial mass and metabolic activities, including citric synthase activity, ATP production, oxygen consumption and mitochondrial complex I activity. Therefore, this novel finding further supported that adipocyte-derived autotaxin was a critical adipokine in modulating cardiac function in obese mice.

In conclusion, present study unraveled a novel crosstalk between adipose tissue and heart. Autotaxin, as an adipokine, deteriorated cardiac structure and function in obese mice, whereas blockage of adipose tissue autotaxin effectively improved cardiac injuries. These novel findings supported that adipose tissue-derived autotaxin was a potential clinical marker for diagnosis and therapeutic target in alleviating obesity-related cardiomyopathy.

\section{Declaration of interest}

The authors declare that there is no conflict of interest that could be perceived as prejudicing the impartiality of the research reported.

\section{Funding}

This work was financially supported from the National Key R\&D Program of China (2016YFC1301100) and National Natural Science Foundation of China (81800269 and 81800362).

\section{References}

Alpert MA, Lavie CJ, Agrawal H, Aggarwal KB \& Kumar SA 2014 Obesity and heart failure: epidemiology, pathophysiology, clinical manifestations, and management. Translational Research 164 345-356. (https://doi.org/10.1016/j.trsl.2014.04.010)

Bouchareb R, Mahmut A, Nsaibia MJ, Boulanger MC, Dahou A, Lepine JL, Laflamme MH, Hadji F, Couture C, Trahan S, et al. 2015 Autotaxin derived from lipoprotein(a) and valve interstitial cells promotes inflammation and mineralization of the aortic valve. Circulation 132 677-690. (https://doi.org/10.1161/ CIRCULATIONAHA.115.016757)

Boucher J, Quilliot D, Praderes JP, Simon MF, Gres S, Guigne C, Prevot D, Ferry G, Boutin JA, Carpene C, et al. 2005 Potential involvement of adipocyte insulin resistance in obesity-associated up-regulation of adipocyte lysophospholipase D/autotaxin expression. Diabetologia 48 569-577. (https://doi.org/10.1007/ s00125-004-1660-8)

Chen Q \& Lesnefsky EJ 2015 Heart mitochondria and calpain 1: location, function, and targets. Biochimica and Biophysica Acta 1852 2372-2378. (https://doi.org/10.1016/j.bbadis.2015.08.004)

DiPietro L, Katz LD \& Nadel ER 1999 Excess abdominal adiposity remains correlated with altered lipid concentrations in healthy older women. International Journal of Obesity and Related Metabolic Disorders 23 432-436. (https://doi.org/10.1038/sj.ijo.0800848)

D'Souza K, Kane DA, Touaibia M, Kershaw EE, Pulinilkunnil T \& Kienesberger PC 2017 Autotaxin is regulated by glucose and insulin in adipocytes. Endocrinology 158 791-803. (https://doi.org/10.1210/ en.2017-00035)

D'Souza K, Nzirorera C, Cowie AM, Varghese GP, Trivedi P, Eichmann TO, Biswas D, Touaibia M, Morris AJ, Aidinis V, et al. 2018 Autotaxin-LPA signaling contributes to obesity-induced insulin resistance in muscle and impairs mitochondrial metabolism. Journal of Lipid Research 59 1805-1817. (https://doi.org/10.1194/jlr.M082008)

Ferry G, Tellier E, Try A, Gres S, Naime I, Simon MF, Rodriguez M, Boucher J, Tack I, Gesta S, et al. 2003 Autotaxin is released from adipocytes, catalyzes lysophosphatidic acid synthesis, and activates preadipocyte proliferation. Up-regulated expression with adipocyte differentiation and obesity. Journal of Biological Chemistry 278 18162-18169. (https://doi.org/10.1074/jbc.M301158200)

Frisard M \& Ravussin E 2006 Energy metabolism and oxidative stress: impact on the metabolic syndrome and the aging process. Endocrine 29 27-32. (https://doi.org/10.1385/ENDO:29:1:27)

Gautron L \& Elmquist JK 2011 Sixteen years and counting: an update on leptin in energy balance. Journal of Clinical Investigation 121 2087-2093. (https://doi.org/10.1172/JCI45888) 
Halberg N, Wernstedt-Asterholm I \& Scherer PE 2008 The adipocyte as an endocrine cell. Endocrinology and Metabolism Clinics of North America 37 753-768, x-xi. (https://doi.org/10.1016/j.ecl.2008.07.002)

Hilal-Dandan R, Means CK, Gustafsson AB, Morissette MR, Adams JW, Brunton LL \& Heller Brown J 2004 Lysophosphatidic acid induces hypertrophy of neonatal cardiac myocytes via activation of Gi and Rho. Journal of Molecular and Cellular Cardiology 36 481-493. (https:// doi.org/10.1016/j.yjmcc.2003.12.010)

Hirst J 2013 Mitochondrial complex I. Annual Review of Biochemistry 82 551-575. (https://doi.org/10.1146/annurevbiochem-070511-103700)

Hubert HB, Feinleib M, McNamara PM \& Castelli WP 1983 Obesity as an independent risk factor for cardiovascular disease: a 26-year follow-up of participants in the Framingham Heart Study. Circulation 67 968-977. (https://doi.org/10.1161/01.CIR.67.5.968)

Janssen I, Katzmarzyk PT \& Ross R 2002 Body mass index, waist circumference, and health risk: evidence in support of current National Institutes of Health guidelines. Archives of Internal Medicine 162 2074-2079. (https://doi.org/10.1001/archinte.162.18.2074)

Kawano H, Do YS, Kawano Y, Starnes V, Barr M, Law RE \& Hsueh WA 2000 Angiotensin II has multiple profibrotic effects in human cardiac fibroblasts. Circulation 101 1130-1137. (https://doi. org/10.1161/01.CIR.101.10.1130)

Kurth T, Gaziano JM, Berger K, Kase CS, Rexrode KM, Cook NR, Buring JE \& Manson JE 2002 Body mass index and the risk of stroke in men. Archives of Internal Medicine 162 2557-2562. (https://doi. org/10.1001/archinte.162.22.2557)

Liu J, Wang F, Xie M \& Chen R 2018 Response to inhibition of TGFbeta1 might be a novel therapeutic target in the treatment of cardiac fibrosis. International Journal of Cardiology 256 20. (https://doi. org/10.1016/j.ijcard.2017.08.064)

Marin-Garcia J, Goldenthal MJ \& Moe GW 2001 Abnormal cardiac and skeletal muscle mitochondrial function in pacing-induced cardiac failure. Cardiovascular Research 52 103-110. (https://doi.org/10.1016/ s0008-6363(01)00368-6)

Nakatsuka A, Wada J, Iseda I, Teshigawara S, Higashio K, Murakami K, Kanzaki M, Inoue K, Terami T, Katayama A, et al. 2013 Visceral adipose tissue-derived serine proteinase inhibitor inhibits apoptosis of endothelial cells as a ligand for the cell-surface GRP78/voltagedependent anion channel complex. Circulation Research 112 771-780. (https://doi.org/10.1161/CIRCRESAHA.111.300049)

Nie H, Song C, Wang D, Cui S, Ren T, Cao Z, Liu Q, Chen Z, Chen X \& Zhou Y 2017 MicroRNA-194 inhibition improves dietary-induced non-alcoholic fatty liver disease in mice through targeting on FXR. Biochimica et Biophysica Acta: Molecular Basis of Disease 1863 3087-3094. (https://doi.org/10.1016/j.bbadis.2017.09.020)

Nie H, Pan Y \& Zhou Y 2018 Exosomal microRNA-194 causes cardiac injury and mitochondrial dysfunction in obese mice. Biochemical and Biophysical Research Communications 503 3174-3179. (https://doi. org/10.1016/j.bbrc.2018.08.113)

Ninou I, Magkrioti C \& Aidinis V 2018 Autotaxin in pathophysiology and pulmonary fibrosis. Frontiers in Medicine 5 180. (https://doi. org/10.3389/fmed.2018.00180)

Nishimura S, Nagasaki M, Okudaira S, Aoki J, Ohmori T, Ohkawa R, Nakamura K, Igarashi K, Yamashita H, Eto K, et al. 2014 ENPP2 contributes to adipose tissue expansion and insulin resistance in diet-induced obesity. Diabetes 63 4154-4164. (https://doi. org/10.2337/db13-1694)
Piche ME, Poirier P, Lemieux I \& Despres JP 2018 Overview of epidemiology and contribution of obesity and body fat distribution to cardiovascular disease: an update. Progress in Cardiovascular Diseases 61 103-113. (https://doi.org/10.1016/j.pcad.2018.06.004)

Rancoule C, Dusaulcy R, Treguer K, Gres S, Guigne C, Quilliot D, Valet P \& Saulnier-Blache JS 2012 Depot-specific regulation of autotaxin with obesity in human adipose tissue. Journal of Physiology and Biochemistry 68 635-644. (https://doi.org/10.1007/s13105-012-0181-z)

Rancoule C, Dusaulcy R, Treguer K, Gres S, Attane C \& SaulnierBlache JS 2014 Involvement of autotaxin/lysophosphatidic acid signaling in obesity and impaired glucose homeostasis. Biochimie 96 140-143. (https://doi.org/10.1016/j.biochi.2013.04.010)

Reeves VL, Trybula JS, Wills RC, Goodpaster BH, Dube JJ, Kienesberger PC \& Kershaw EE 2015 Serum autotaxin/ENPP2 correlates with insulin resistance in older humans with obesity. Obesity 23 2371-2376. (https://doi.org/10.1002/oby.21232)

Shetty S, Kusminski CM \& Scherer PE 2009 Adiponectin in health and disease: evaluation of adiponectin-targeted drug development strategies. Trends in Pharmacological Sciences 30 234-239. (https://doi. org/10.1016/j.tips.2009.02.004)

Shibata R, Sato K, Pimentel DR, Takemura Y, Kihara S, Ohashi K, Funahashi T, Ouchi N \& Walsh K 2005 Adiponectin protects against myocardial ischemia-reperfusion injury through AMPK- and COX-2dependent mechanisms. Nature Medicine 11 1096-1103. (https://doi. org/10.1038/nm1295)

Sun S, Wang R, Song J, Guan M, Li N, Zhang X, Zhao Z \& Zhang J 2017 Blocking gp130 signaling suppresses autotaxin expression in adipocytes and improves insulin sensitivity in diet-induced obesity. Journal of Lipid Research 58 2102-2113. (https://doi.org/10.1194/jlr. M075655)

Takeishi Y, Niizeki T, Arimoto T, Nozaki N, Hirono O, Nitobe J, Watanabe T, Takabatake N \& Kubota I 2007 Serum resistin is associated with high risk in patients with congestive heart failure - a novel link between metabolic signals and heart failure. Circulation Journal 71 460-464. (https://doi.org/10.1253/circj.71.460)

Tamura N, Ogawa Y, Chusho H, Nakamura K, Nakao K, Suda M, Kasahara M, Hashimoto R, Katsuura G, Mukoyama M, et al. 2000 Cardiac fibrosis in mice lacking brain natriuretic peptide. PNAS 97 4239-4244. (https://doi.org/10.1073/pnas.070371497)

Tamura T, Furukawa Y, Taniguchi R, Sato Y, Ono K, Horiuchi H, Nakagawa Y, Kita T \& Kimura T 2007 Serum adiponectin level as an independent predictor of mortality in patients with congestive heart failure. Circulation Journal 71 623-630. (https://doi.org/10.1253/ circj.71.623)

Yang J, Xu J, Han X, Wang H, Zhang Y, Dong J, Deng Y \& Wang J 2018 Lysophosphatidic acid is associated with cardiac dysfunction and hypertrophy by suppressing autophagy via the LPA3/AKT/mTOR pathway. Frontiers in Physiology 9 1315. (https://doi.org/10.3389/ fphys.2018.01315)

Yuelling LM \& Fuss B 2008 Autotaxin (ATX): a multi-functional and multi-modular protein possessing enzymatic lysoPLD activity and matricellular properties. Biochimica and Biophysica Acta 1781 525-530. (https://doi.org/10.1016/j.bbalip.2008.04.009)

Zhou M, Bao Y, Li H, Pan Y, Shu L, Xia Z, Wu D, Lam KS, Vanhoutte PM, Xu A, et al. 2015 Deficiency of adipocyte fatty-acidbinding protein alleviates myocardial ischaemia/reperfusion injury and diabetes-induced cardiac dysfunction. Clinical Science 129 547-559. (https://doi.org/10.1042/CS20150073)

Received in final form 13 June 2019

Accepted 18 June 2019

Accepted Preprint published online 18 June 2019 (c) 2019 Society for Endocrinology Published by Bioscientifica Ltd. Printed in Great Britain 